Case report

\title{
Pituitary macroadenoma co-existent with supraclinoid internal carotid artery cerebral aneurysm: a case report and review of the literature Chia-Sheng Wang ${ }^{1}$, Tsung-Chih Yeh ${ }^{1}$, Tai-Ching $\mathrm{Wu}^{2}$ and
Chao-Hung $\mathrm{Yeh}^{1 *}$
}

Addresses: ${ }^{1}$ Department of Neurosurgery, Chi-Mei Medical Center, 901 Chung Hwa Road, Yung Kang City, Tainan, Taiwan 710

${ }^{2}$ Department of Radiology, Chi-Mei Medical Center, 901 Chung Hwa Road, Yung Kang City, Tainan, Taiwan 710

Email: C-SW - cswang17@yahoo.com.tw; TCY - chimeins@gmail.com; TCW - tcwu7520@gmail.com; CHY* - nsyeh5855@gmail.com

* Corresponding author

Received: 8 March 2009 Accepted: 5 June 2009 Published: 23 July 2009

Cases Journal 2009, 2:6459 doi: 10.4076/1757-1626-2-6459

This article is available from: http://casesjournal.com/casesjournal/article/view/6459

(C) 2009 Wang et al.; licensee Cases Network Ltd.

This is an Open Access article distributed under the terms of the Creative Commons Attribution License (http://creativecommons.org/licenses/by/3.0),

which permits unrestricted use, distribution, and reproduction in any medium, provided the original work is properly cited.

\begin{abstract}
With improved angiographic techniques and magnetic resonance angiography available today, an increasing number of incidental aneurysms are being detected. Occurrence of an intracranial aneurysm together with a pituitary adenoma presents tremendous risk to the patient, particularly when the aneurysm lies near the operative field.
\end{abstract}

A 6I-year-old woman presented with a progressive visual field defect. Neurological examination revealed bi-temporal haemianopia. Cerebral magnetic resonance imaging and angiography revealed a pituitary macroadenoma co-existent with a cerebral aneurysm near the sellar region. The patient underwent an endovascular procedure for aneurysm embolisation and then underwent surgery for removal of the pituitary adenoma via a trans-sphenoidal approach.

We report our experience and emphasize the need for critical evaluation of neuroradiological examinations for precise diagnosis for avoiding a possible life-threatening situation.

\section{Introduction}

The possible co-existence of an intracranial aneurysm and a pituitary adenoma has been previously reported in the literature $[1,2,3]$. With improved angiographic techniques and MR angiography available today, an increasing number of incidental aneurysms are being detected. Occurrence of an intracranial aneurysm together with a pituitary adenoma presents tremendous risk to the patient, particularly when the aneurysm lies near the operative field [4]. Because of invasion of the pituitary region, an intracranial aneurysm can sometimes mimic a sellar lesion. We report a 61-year-old woman with a pituitary macroadenoma co-existent with a cerebral aneurysm near the sellar region. Since such cases are possible life-threatening situations, the clinical presentation and management are discussed here.

\section{Case presentation}

A 61-year-old Asian Taiwanese woman presented to our institution with a progressive visual field defect. She had 
a history of hypertension for 20 years. Neurological examination revealed bi-temporal haemianopia. She also complained of occasional headache associated with nausea, vomiting and vertigo. Hormonal studies showed a slight elevation in prolactin levels. A brain computed tomography (CT) scan showed a slightly hyperdense lesion in the sellar region and a partially eroded sellar floor. Cerebral magnetic resonance imaging (MRI) revealed an expanding lesion inside the sella with suprasellar extension (Figure 1). On MRI, a flow void mass in the right supraclinoid internal carotid artery (ICA) raised the suspicion of a cerebral aneurysm (Figure 2). Cerebral angiography confirmed the presence of a wide-neck saccular-type aneurysm (measuring approximately $8.0 \times$ $7.5 \mathrm{~mm}$; neck, $4.8 \mathrm{~mm}$ ) in the right supraclinoid ICA, pointing superiorly with a small side lobe near its neck which pointed laterally (Figure 3A). Initially, we treated the aneurysm by endovascular coil placement (Figure 3B). One month later, the patient underwent surgical decompression of the sella and excision of the tumour via a transnasal trans-sphenoidal endoscopic approach. Subtotal resection of the adenoma was performed, and the suprasellar portion was preserved to lower any possible risk to the coil-treated aneurysm. Histological examination confirmed the diagnosis of a prolactin-secreting adenoma. The patient's postoperative course was uneventful, and the post-operative assessment of visual acuity, visual fields and extra-ocular movements showed no significant changes from the pre-operative assessment. The post-operative pituitary hormonal status was normal. The patient underwent

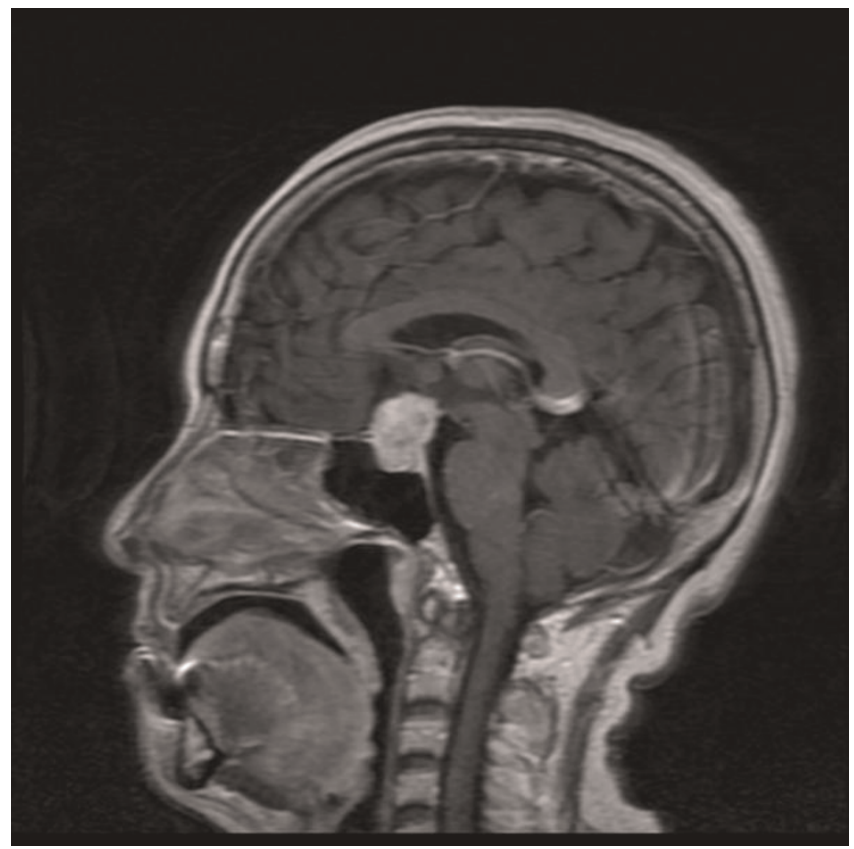

Figure I. Gadolinium-enhanced sagittal MRI showing an expanding lesion inside the sella with suprasellar extension.

adjuvant X-knife radiotherapy for the residual suprasellar portion of the adenoma. At 1-year follow-up, the patient had no neurological deficits, and MRI showed a normal pituitary gland and stalk.
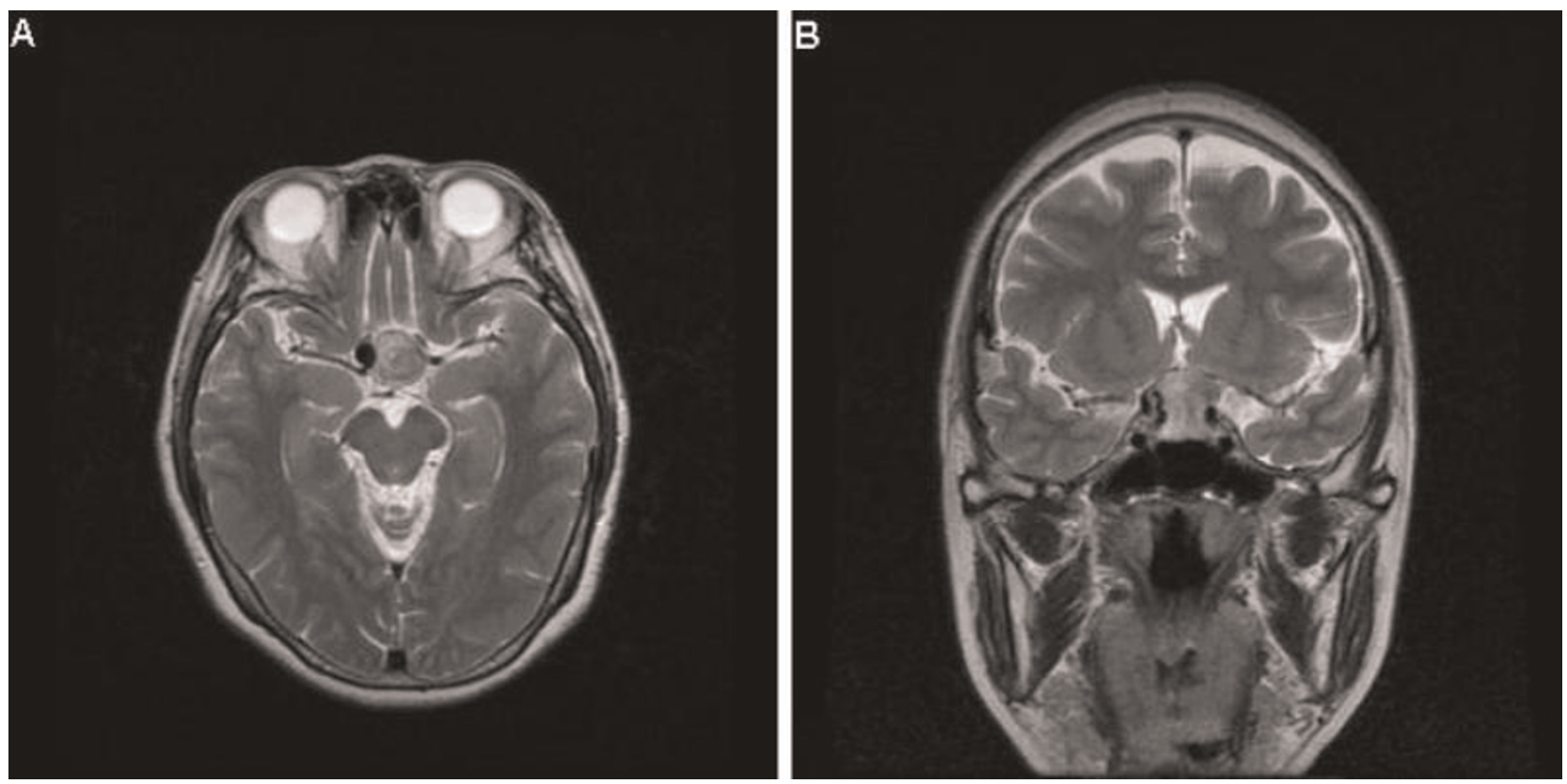

Figure 2. Axial (A) and Coronal (B) view of T2-weighted MRI showing a round flow-void mass in the right supraclinoid ICA associated with a pituitary macroadenoma. 

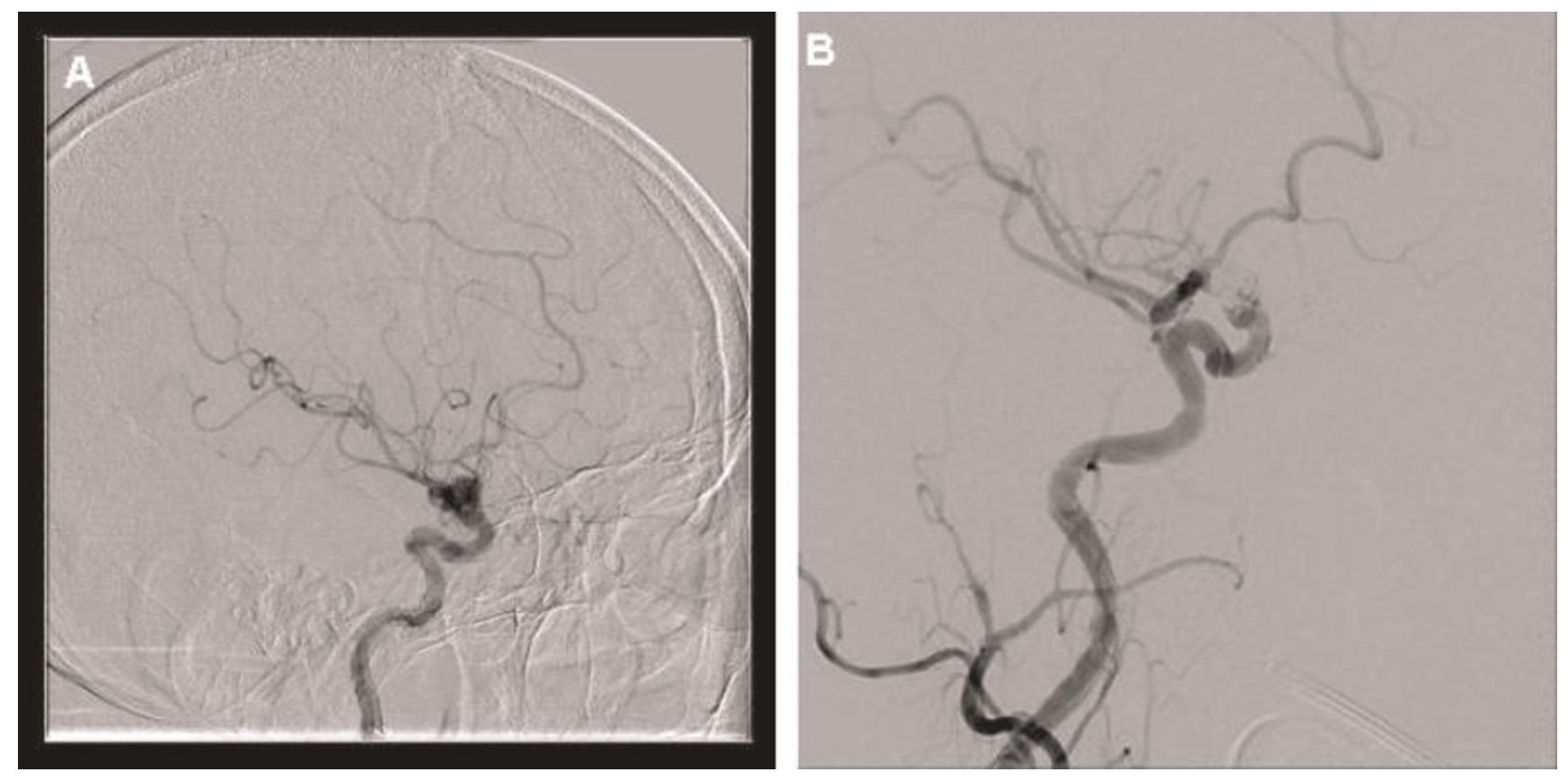

Figure 3. (A) Right internal carotid angiogram showing a wide-neck saccular-type aneurysm (measuring approximately $8 \times 7.5 \mathrm{~mm}$; neck, $4.8 \mathrm{~mm}$ ) in the right supraclinoid ICA, pointing superiorly and with a small side lobe near its neck, pointing laterally. (B) Right internal carotid angiogram obtained after endovascular coil placement. The aneurysm has been completely obliterated.

\section{Discussion}

In this report, a 61-year-old woman was diagnosed of a pituitary macroadenoma co-existent with a cerebral aneurysm near the sellar region.

Hypotheses for this phenomenon have been proposed, which include a direct mechanical effect of the pituitary adenoma on the vasculature [5], circulatory changes due to involvement of the skull base [6], direct infiltration by the tumour [7], and growth hormone production leading to arteriosclerotic and degenerative changes in the arterial walls of the circle of Willis $[8,9]$, predisposing to aneurysm formation or enlargement.

The incidence of intracranial aneurysms in the general population is now considered to be around 5\% [4,10-12]. The consensus is that the incidence of an intracranial aneurysm associated with pituitary adenoma is low. However, in the clinical series reported by Wakai et al. [9], the incidence of associated aneurysms with 95 pituitary tumours was $7.4 \%$. In another retrospective study of 467 cases of pituitary adenomas, the incidence of associated intracranial aneurysms was 5.4\% [4]. Thus, such association has been reported to range from $3.7 \%$ to $7.4 \%[4,13]$.

According to the previous review by Locatelli et al. [1], an intracranial aneurysm that co-exists with a sellar lesion is more frequently observed in patients with aneurysms of the ICA and the anterior communicating artery because they supply the pituitary region. In one retrospective study [4], 48\% of intracranial aneurysms associated with pituitary adenoma were observed in the internal carotidposterior cerebral artery followed by IC-ophthalmic aneurysms in $19 \%$ and IC bifurcation in 13\%. This distribution pattern resembled that observed in other autopsy series of the normal population [14]. Moreover, $60 \%$ of aneurysms were near the parasellar region and $40 \%$ at distant locations [4].

Different clinical situations affecting the sellar region present with similar neuroradiological features. Pituitary tumour apoplexy, generally caused by acute expansion of an infracted or haemorrhagic pituitary adenoma extending laterally in the cavernous sinus or toward the optic chiasma and optic nerves, is a clinical situation characterized by a sudden onset of severe headaches associated with nausea, vertigo and/or altered mental status. Ophthalmoplegia and deterioration of visual acuity and visual fields are also frequently present. In cases of subarachnoid haemorrhage caused by the rupture of cerebral aneurysm, it may be sometimes difficult to differentiate it from pituitary apoplexy, particularly when the cerebral aneurysm lies near the sellar region. One case report described the thrombosis of a cerebral aneurysm of the intracavernous tract of the carotid artery mimicking apoplexy 
of a pituitary adenoma [1]; another study has reported the co-existence of aneurysmal subarachnoid haemorrhage with pituitary apoplexy [15].

Cerebral aneurysms can be treated by endovascular or microsurgical techniques [16-18]. In the present case, the patient experienced typical symptoms and signs due to the pituitary tumour and optic chiasma compression. With an aim to prevent the possible risk to the patient from the proximity of the aneurysm to the operative field, the cerebral aneurysm was first treated with endovascular coil placement; subsequently, surgical decompression of the sella was performed. The suprasellar portion of the pituitary adenoma was preserved during the trans-sphenoidal procedure. Our treatment plan in this case included initial endovascular embolisation for the cerebral aneurysm, followed by a transnasal trans-sphenoidal endoscopic approach for surgical decompression of the sella and excision of the pituitary adenoma below the diaphragm sella along with adjuvant $\mathrm{X}$-knife radiotherapy for the suprasellar portion of adenoma-a plan that aimed to lower the possible risk to the patient during the interventional procedures.

Our experience reinforces the need for the critical evaluation of neuroradiological examinations. In such situations, the need for precise diagnosis is emphasized to avoid a possible life-threatening situation whenever an interventional approach is considered.

\section{Abbreviations}

MRI, Magnetic Resonance Imaging; CT, Computed Tomography; ICA, Internal Carotid Artery.

\section{Consent}

Written informed consent was obtained from the patient for publication of this case report and accompanying images. A copy of the written consent is available for review by the Editor-in-Chief of this journal.

\section{Competing interests}

The authors declare that they have no competing interests.

\section{Authors' contributions}

$\mathrm{CHY}$ is the attending surgeon and revised the final manuscript. CHY, TCY and TCW carried out the patient diagnosis, investigation, and management. CSW carried out the patient follow up and performed the literature search, general coordination, drafting of the manuscript, writing the final manuscript. All authors read and approved the final manuscript.

\section{References}

I. Locatelli M, Spagnoli D, Caroli M, Isalberti M, Branca V, Gaini SM, Lania A: A potential catastrophic trap: an unusually presenting sellar lesion. Eur J Neurol 2008, 15:98-10I.
2. Handa J, Matsuda I, Handa H: Association of brain tumor and intracranial aneurysms. Surg Neurol 1976, 6:25-29.

3. Weir B: Pituitary tumors and aneurysms: case report and review of literature. Neurosurgery 1992, 30:585-59I.

4. Pant B, Arita K, Kurisu K, Tominaga A, Eguchi K, Uozumi T: Incidence of intracranial aneurysm associated with pituitary adenoma. Neurosurg Rev 1997, 20:13-17.

5. Jakubowski J, Kendall B: Coincidental aneurysms with tumors of pituitary origin. J Neurol Neurosurg Psychiatry 1978, 41:972-979.

6. Du Boulay GH: Some observations on the natural history of intracranial aneurysms. Br J Radiol 1965, 38:72I-757.

7. Mangiardi JR, Aleksic SN, Lifshitz M, Pinto R, Budzilovic GN, Pearson J: Coincidental pituitary adenoma and cerebral aneurysm with pathological findings. Surg Neurol 1983, 19:38-4I.

8. Bar RS, Boes M, Dake BL, Booth BA, Henley SA, Sandra A: Insulin, insulin-like growth factors, and vascular endothelium. Am J Med 1988, 85:59-70.

9. Wakai S, Fukushima T, Furihata T, Sano K: Association of cerebral aneurysm with pituitary adenoma. Surg Neurol 1979, 12:503-507.

10. McCormick WF, Acosta-Rua G]: The size of intracranial saccular aneurysms. J Neurosurg 1970, 33:422-427.

II. Nakagawa $T$, Hashi $K$ : The incidence and treatment of asymptomatic, unruptured cerebral aneurysms. I Neurosurg 1994, 80:217-223.

12. Sekhar LN, Heros RC: Origin, growth, and rupture of saccular aneurysms: a review. Neurosurgery 198I, 8:248-260.

13. Acqui M, Ferrante L, Fraioli B, Cosentino F, Fortuna A, Mastronardi L: Association between intracranial aneurysms and pituitary adenomas. A etiopathogenetic hypotheses. Neurochirurgia (Stuttg) 1987, 30:177-181.

14. Housepian EM, Pool JL: A systematic analysis of intracranial aneurysms from the autopsy file of the Presbyterian Hospital, 1914 to 1956. J Neuropathol Exp Neurol 1958, 17:409-423.

15. Laidlaw JD, Tress B, Gonzales MF, Wray AC, Nq WH, O'Brien JM: Coexistence of aneurysmal subarachnoid haemorrhage and pituitary apoplexy: Case report and review of the literature. J Clin Neurosci 2003, 10:478-482.

16. Salpietro FM, Longo M, Alafaci C, Gervasio O, Tomasello F: Co-existing pituitary tumour and intracavernous asymptomatic aneurysm: management implications. Acta Neurochir (Wien) 1997, |39:79|-792.

17. Sade B, Mohr G, Tampieri D, Rizzo A: Intrasellar aneurysm and a growth hormone-secreting pituitary macroadenoma. J Neurosurg 2004, 100:557-559.

18. Seda L Jr, Cukiert A, Nogueira KC, Huayllas MK, Liberman B: Intrasellar internal carotid aneurysm coexisting with GH-secreting pituitary adenoma in an acromegalic patient. Arq Neuropsiquiatr 2008, 66:99-100.

\section{Do you have a case to share?}

\author{
Submit your case report today \\ - Rapid peer review \\ - Fast publication \\ - PubMed indexing \\ - Inclusion in Cases Database
}

\section{Any patient, any case, can teach us something}

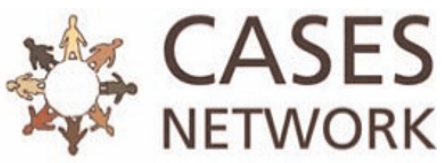

www.casesnetwork.com 